\title{
THE EFFECTS OF RE, W AND RU ON MICROSEGREGATION BEHAVIOUR IN SINGLE CRYSTAL SUPERALLOY SYSTEMS
}

\author{
R.M. Kearsey ${ }^{1}$, J.C. Beddoes ${ }^{2}$, K.M. Jaansalu ${ }^{3}$, W.T. Thompson ${ }^{3}$, P.Au ${ }^{1}$ \\ ${ }^{1}$ Institute for Aerospace Research, National Research Council of Canada, Ottawa, ON, Canada \\ ${ }^{2}$ Department of Mechanical and Aerospace Engineering, Carleton University, Ottawa, ON, Canada \\ ${ }^{3}$ Department of Chemistry and Chem. Engineering, Royal Military College of Canada, Kingston, ON, Canada
}

Keywords: microsegregation, single crystal, Bridgman, partitioning, computational thermodynamics, EPMA, DSC.

\begin{abstract}
The major advancements in mechanical and thermal properties of the most recently developed single crystal (SX) superalloys can be attributed to the addition of specific refractory elements into base alloy compositions. The present study investigates the effect of modifying refractory addition levels on the solidification behaviour of SX superalloys systems. Specifically, a series of six Ni-base alloy compositions are set in a controlled manner, such that the chemical microsegregation effects of Re, W, and Ru can be independently assessed. Fabrication of grain-free SX bars from each alloy composition is achieved by utilizing a modified Bridgman casting process, with subsequent compositional analysis of the solidification structures via electron microprobe analysis (EPMA) methods. Further validation of these EPMA microsegregation results are supported by means of eutectic phase fraction analysis and differential scanning calorimetry (DSC) methods. Qualitative partitioning results indicate typical SX alloy segregation behavior with elements such as $\mathrm{Cr}, \mathrm{Co}, \mathrm{Re}, \mathrm{Mo}$, and $\mathrm{W}$ all segregating towards the dendrite core regions, while the $\gamma^{\prime}$ forming elements of $\mathrm{Al}, \mathrm{Ti}$, and $\mathrm{Ta}$ partition to the interdendritic $\gamma-\gamma^{\prime}$ eutectic regions. Both $\mathrm{Ni}$ and $\mathrm{Ru}$ exhibit ideal segregation behaviour with no favorable partitioning to either liquid or solid phase. Quantitative EPMA results indicate that as the nominal Re level increases, the severity of microsegregation to the dendrite core regions rises dramatically for $\mathrm{Mo}, \mathrm{Cr}$, and $\mathrm{Re}$. Evidence is presented that demonstrates the role that Ru plays in counteracting the microsegregation effects of both increased $\mathrm{Re}$ and higher overall total refractory levels. In addition to experimental trials, chemical partitioning predictions are also presented for the alloy system, utilizing a solid-liquid phase equilibria model generated using a customized chemical thermodynamic database. Using this CALPHAD approach, a comparison of the computational predictions and the actual experimental segregation results is also provided for discussion.
\end{abstract}

\section{Introduction}

The next generation of advanced fighters and transport aircraft stipulates the use of advanced SX superalloys for critical turbine components to accommodate peak performance demands. However, SX technology is not mature, as the relationships between alloy composition, processing parameters, microstructure, phasial/environmental stability, and mechanical properties are not fully understood and require additional characterization [1]. Perhaps one of the most difficult tasks of a turbine blade manufacturer is the selection of a suitable alloy composition that is capable of withstanding the arduous environment of the gas turbine engine. Selection of a suitable alloy composition must be made on the basis of both mechanical and microstructural stability over extended periods of high temperature application. Advancement in mechanical and thermal properties of SX superalloy blades has been primarily attributed to the increased addition of specific refractory elements into base alloy compositions. In particular, numerous studies have shown rhenium additions to be useful for optimizing thermal-mechanical properties for many of the SX Ni-base superalloys [2,3,4,5]. Although the improvements in strength and high temperature capability are desirable, as the overall level of refractory additions is increased, so does the degree of non-homogeneous chemical distribution within the alloy microstructure.

In general, most solidification processes result in some degree of non-homogeneous chemical distribution due to naturally occurring mass transport phenomena. The degree or severity of segregation within most cast alloys is usually dependent on one or more of the following variables:

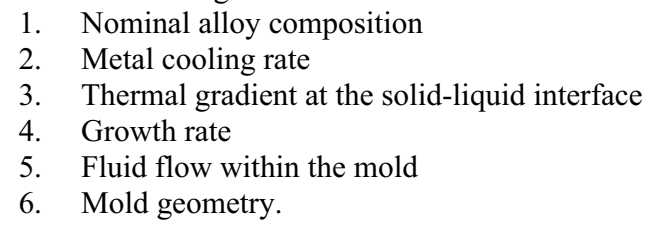

Complex directional solidification processes, such as Bridgman or Czochralski methods, utilize a narrow range of specific casting parameters required to yield defect-free, homogeneous products. Hence, casting experimentation and/or predictive solidification modeling are required to determine suitable casting parameters necessary to control the influence of each of these variables on both microsegregation and macrosegregation.

Microsegregation results from the uneven mass transport phenomenon that occurs on a microscopic level during solidification at the solid-liquid interface. The localized, nonuniform chemical distribution that occurs is due to the rejection of solute atoms by the recently formed solid phase into the more diffusive interdendritic liquid region [6]. This produces a localized compositional gradient across the dendritic solidification structure of the alloy, ranging from the initial dendrite core region to the final interdendritic liquid phase that solidifies. Furthermore, depending on the casting parameters used and the number of diffusing solute species (i.e. alloying additions), this compositional gradient can change gradually across the $\sim 150$ to $250 \mu \mathrm{m}$ segregation zone, or a major inflection in the chemical gradient may result. The first case is the desired casting result that can usually be easily homogenized with post-casting heat treatments. The latter case can result in major changes in microstructural constituents due to severe microsegregation. In 
fact, it is not uncommon to see eutectic phase formation during the final stages of solidification due to the favorable partitioning of eutectic forming species in the final interdendritic region [7]. The severity of microsegregation can be quantified by measuring the difference in composition between the dendrite cores and the interdendritic regions by performing EPMA. In addition to quantifying the degree of chemical partitioning between phases, the extent of microsegregation may also be gauged based on the fraction of eutectic phase that is formed. In general, the greater the fraction of eutectic phase present in the as-cast microstructure, the greater the degree of microsegregation. Therefore, it is important to quantify both the degree of chemical partitioning and the phase fraction of eutectic in the cast alloy in order to characterize the severity of microsegregation. Depicted in Figure 1 is an exaggerated representation of the microsegregation pattern typically observed in most as-cast SX superalloys. Highlighted are the concentrated levels of $\mathrm{Re}, \mathrm{Cr}, \mathrm{Mo}, \mathrm{W}, \mathrm{Co}$ at the dendrite cores and elevated levels of $\mathrm{Al}, \mathrm{Ti}$ and $\mathrm{Ta}$ within the interdendritic regions.

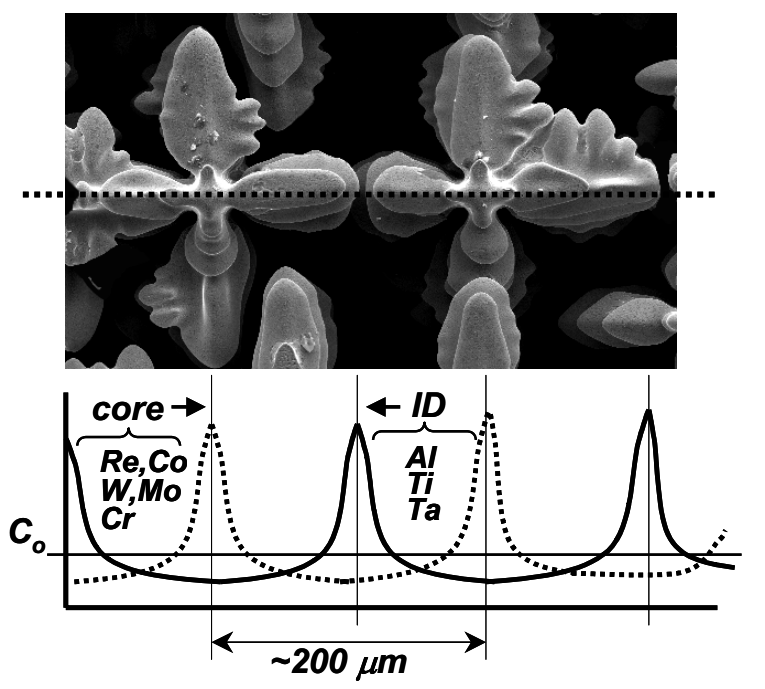

Figure 1: Representation of the microsegregation pattern typical in most as-cast SX superalloys illustrating preferential partitioning of specific refractory elements to the core and $\gamma^{\prime}$ elements to interdendritic (ID) regions [8].

An undesirable by-product of these localized concentrations of solute atoms is the increased tendency for the formation of deleterious topologically close-packed (TCP) phases [9]. These phases degrade the mechanical properties of SX Ni-base superalloys, counteracting the original purpose for increasing refractory levels [10]. For this reason, the severity of microsegregation as a function of individual and overall alloy addition levels is a vital design factor for SX casting vendors and turbine blade manufacturers. For the most part, completely avoiding the formation of these deleterious phases has proven to be almost impossible; hence, the main objective has been to reduce the fraction of TCP phase formation at various high temperatures and to delay the onset of nucleation. This is achieved by carefully selecting alloy compositions such that a uniform duplex $\gamma-\gamma^{\prime}$ microstructure is easily attained via standard homogenization heat treatments. In general, alloy compositions that yield less as-cast microsegregation are more easily homogenized and result in delayed TCP phase nucleation and growth kinetics. The end results are less-complex furnace heat treatment processes and extended microstructural stability lifetimes for gas turbine components.

The main objective of this study was to investigate the compositional effects of various alloying additions on chemical microsegregation. A short explanation of the composition selection and casting process is provided. The main characterization methods used to measure the severity of microsegregation are presented; specifically, the EPMA quantitative analysis, eutectic phase fraction analysis, and DSC thermo-physical results. The effects of $\mathrm{Re}, \mathrm{Ru}$ and $\mathrm{W}$ on microsegregation are discussed based on these results. A comparative study is also presented, based on the experimental EPMA microsegregation values and the theoretical CALPHAD predictions generated using the multi-component thermodynamic model [11].

\section{Experimental Materials}

As with any complex alloy system, the ability to precisely relate changes in material properties to individual or even combinations of elemental additions is extremely difficult to achieve [12]. This is due to the unpredictable binary, ternary, and higher order synergistic effects present in these highly alloyed systems that may contain in excess of ten or more elemental species in nominal alloy compositions. In addition to having this large number of chemical interaction parameters, the uncertainty in quantifying specific chemical effects is even further magnified with the compositional variation inherent within SX microstructures due to selective element partitioning that occurs upon solidification. Although grain boundary segregation is not an issue, the distinctive solidification structure that is produced in these SX alloys during the slow casting process results in non-uniform chemical distribution between the dendritic and interdendritic regions. Therefore, for the purpose of this study, it was necessary to ascertain a series of experimental alloy compositions that could be used for comparison testing to differentiate the effects of specific alloying additions. Furthermore, it was also necessary to determine which elements would be of prime importance for industrial designers for present and future blade alloy applications.

Through the development of new SX alloy design initiatives, researchers have indirectly determined the independent effects of Re on phase stability and high temperature creep properties [13]. Of particular interest to material designers are the synergistic effects of $\mathrm{Re}$ and $\mathrm{W}$, which are known to participate in topologically close-packed (TCP) phase formation [10]. Prevention of TCP phase formation is critical, as the performance of these alloys depreciates with increasing TCP volume fraction. More recently, some of the $4^{\text {th }}$ generation of SX superalloys (e.g. MC-NG) have reported improvements in microstructural stability while decreasing overall alloy density due to the addition of $\mathrm{Ru}$ into nominal alloy compositions [14]. Hence, the selection of a series of experimental alloys was determined based on two major requirements: 1) the compositions must yield a full range of phase-stability/chemical partitioning properties required for comparison purposes, and 2) the alloy compositions must be set in a controlled manner such that changes in chemical segregation can be directly attributed to the specific elemental additions of $\mathrm{Re}$, $\mathrm{W}$, and $\mathrm{Ru}$. With these criteria in mind, the target alloy compositions listed in Table I were selected. 
Table I: Chemical composition, total refractory level, and alloy density for experimental alloys.

\begin{tabular}{lccccccc}
\hline Alloy & \multicolumn{1}{c}{ Chemical composition* } & (wt.\%) & & & \multicolumn{2}{c}{ Total refractory (wt.\%) } & Alloy density \\
\cline { 2 - 8 } & $\mathrm{Ni}$ & $\mathrm{Ta}$ & $\mathrm{W}$ & $\mathrm{Re}$ & $\mathrm{Ru}$ & $\mathrm{W}+\mathrm{Ta}+\mathrm{Co}+\mathrm{Re}+\mathrm{Ru}$ & 21 \\
\hline Alloy A & 63.5 & 12 & 3 & 1 & 0 & 21 & 9.77 \\
Alloy B & 63.5 & 12 & 2 & 2 & 0 & 21 & 9.78 \\
Alloy C & 63.5 & 12 & 1 & 3 & 0 & 21 & 9.80 \\
Alloy D & 63.5 & 12 & 0 & 4 & 0 & 24 & 9.81 \\
Alloy E & 60.5 & 12 & 4 & 3 & 0 & 24 & 9.11 \\
Alloy F & 60.5 & 11 & 1 & 3 & 4 & & 95 \\
\hline
\end{tabular}

* In addition, each alloy also contains: $7.5 \% \mathrm{Cr}+5 \% \mathrm{Co}+2 \% \mathrm{Mo}+1 \% \mathrm{Ti}+5 \% \mathrm{Al}$.

Alloy A through Alloy D represent a complete range of compositional variance with respect to $\mathrm{W}$ and $\mathrm{Re}$ while maintaining a constant total refractory level of $21 \mathrm{wt} . \%$. The composition of Alloy E was specifically chosen to examine the synergistic effects of high $\mathrm{W}$ and $\mathrm{Re}$ addition levels. The composition of Alloy F was selected based on the new generation of SX superalloys that effectively incorporate substantial $\mathrm{Ru}$ additions to aid in phase stability and to decrease overall alloy density. Also included in Table I are the alloy densities. It should also be mentioned that final as-cast wet analysis results indicated alloy addition levels very close to the desired alloy target levels.

\section{Experimental Procedures}

\section{$\underline{\text { SX Casting Process }}$}

The common industrial practice for casting SX alloys is to use complex ceramic molds that incorporate a pigtail crystal selector design. However, an alternative SX casting method was chosen that implements a seed crystal to nucleate crystal growth. An illustration of the experimental Bridgman-seed casting arrangement is provided in Figure 2.

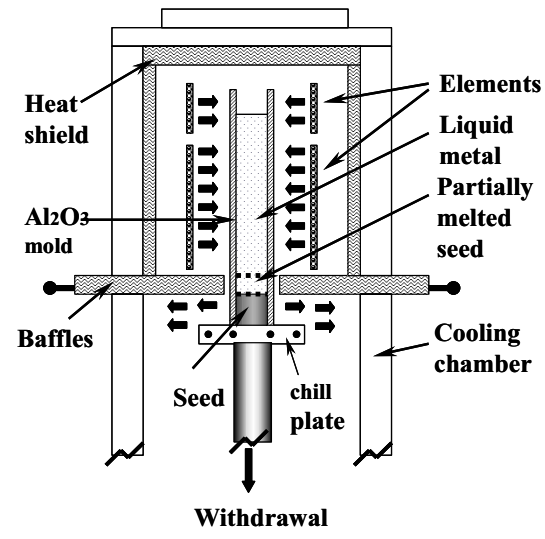

Figure 2: Experimental Bridgman-seed casting setup.

Details of the procedure involve using SX PWA-1484 as starter seed material for the new alloys. In order to promote SX crystal growth in the raw liquid metal, the top $5-10 \mathrm{~mm}$ of the starter seed is positioned partially within the furnace hot-zone and melted by using a superheat temperature of $1585^{\circ} \mathrm{C}$. Once the solid/liquid interface has been established, the ceramic mold containing the partially melted seed/ liquid metal combination is withdrawn from the hot-zone into the cold-zone of the furnace at a rate of $20 \mathrm{~cm} / \mathrm{hr}$ $(8.0 \mathrm{in} / \mathrm{hr}$ ) to prevent SX breakdown. In order to achieve a suitable solid/liquid interface, it was necessary to place a 2-cm $\mathrm{Al}_{2} \mathrm{O}_{3}$ spacer between the seed crystal and chill plate so that an overall thermal gradient of $50{ }^{\circ} \mathrm{C} / \mathrm{cm}$ was obtained. The best castings were also attained when performed under high-vacuum conditions, which prevented major gas entrapment and surface porosity. After casting, it was necessary to examine each $16 \mathrm{~mm}$ (5/8 in.) diameter bar to ensure a SX microstructure throughout its entire $20 \mathrm{~cm}$ ( 8 in.) length.

\section{$\underline{\text { Metallographic Preparation }}$}

As mentioned, each casting required metallographic examination to ensure that a SX structure was obtained. Transverse and longitudinal samples were taken from each bar, polished to a 1$\mu \mathrm{m}$ finish, and etched for 65 seconds in a solution of $10 \mathrm{ml} \mathrm{HCl}$, $10 \mathrm{ml} \mathrm{HNO}_{3}, 0.3 \mathrm{~g}$ molybdic acid, and $15 \mathrm{ml} \mathrm{H}_{2} 0$. This $\gamma^{\prime}$ removing etchant produced suitable image contrast for both optical and SEM microscopy.

In addition to standard metallographic analysis, specimens were also prepared for electron microprobe investigation. The specimens were polished down to a $0.1 \mu \mathrm{m}$ finish and left in an un-etched condition so that precise quantitative analysis results could be acquired. A gold sputter-coat for all EPMA samples produced ideal back-scattered electron imaging results.

\section{Microsegregation Characterization Methods}

\section{EPMA Method}

In order to determine the degree of microsegregation present within the dendrite core and interdendritic $\gamma-\gamma^{\prime}$ eutectic regions, an accurate compositional analysis was required for each alloy. This was achieved using a Camebax MBX Electron Microprobe equipped with 4 WDX spectrometers. Multiple WDX point scans on representative sample areas were performed to obtain a better statistical representation of the different localized compositions. No less than 20 WDX scans were performed for each alloy, 10 within the dendrite core regions and 10 within the interdendritic regions. These point analyses were executed from multiple locations on the sample surface. During X-ray sampling, the probe was operated at $20 \mathrm{kV}$ accelerating potential, a beam current of 35 $\mathrm{nA}$, with the beam rastered over a $10 \times 10 \mu \mathrm{m}$ area. Peak counting times for each element were 30 seconds or 40,000 counts. Background counting times were $+/-15$ seconds on each side of the peak. Wavelength scans were made around the peak position of each analyzed element to check for x-ray interferences and to choose optimum (interference-free) background positions. X-ray data were converted to elemental weight percent by the Cameca PAP matrix correction software. A suite of wellcharacterized pure metals and minerals were employed as calibration standards. Extremely accurate quantitative results were obtained due to the high degree of sampling and also because of 
the care taken in determining the limits of background X-ray noise for elements present in trace amounts (e.g. W and Re).

In addition to the extensive number of point scans performed, a series of line scans was also gathered for each alloy in order to determine the change in chemical gradient across the dendritic solidification structure. For each alloy, line scans were performed along both secondary dendrite arm directions as well as diagonally across the dendrite core into the interdendritic regions. This procedure was duplicated to ensure repeatability. Accelerating potential, beam current and spot size equipment settings were the same as for the previously mentioned point scan procedure.

\section{Eutectic Phase Fraction Analysis}

To further elaborate on the effects of microsegregation within the group of experimental alloys, a full analysis of the eutectic phase fraction present in each of the as-cast microstructures was conducted. This was accomplished by analyzing a number of lowmagnification optical images for each alloy. Samples were prepared using traditional metallographic techniques and etched to reveal the dendritic/eutectic solidification structure. The image analysis software, Image Tool ${ }^{\mathrm{TM}}$, was used to process and analyze each image. The procedure involved capturing the optical image, converting it to 16-bit grayscale format, and adjusting the brightness and contrast settings so that the eutectic and dendritic regions were clearly delineated. Once the image was prepared for analysis, a black and white threshold image was generated using an Image Tool ${ }^{\mathrm{TM}}$ subroutine. It was determined that in order to remove any residual pixels due to variation in dendrite colour (contrasting artifact due to precipitate texturing), a $3 \times 3$ median filter was applied to the threshold image. This effectively filled in the dendrites making them solid black in color. In the case of variation in eutectic color, a pixel-editing tool was used to remove any black areas that would be incorrectly counted as dendritic area. This process was performed manually and proved to be very time consuming in the case where high volume fractions of eutectic were present.

\section{DSC Analysis and Thermo-Physical Characterization}

An extensive DSC study was performed for each of the experimental alloy compositions. Representative $\varnothing 3-\mathrm{mm}$ samples $(\sim 100 \mathrm{mg})$ were tested at thermal heating and cooling rates of 20 ${ }^{\circ} \mathrm{C} / \mathrm{min}, 10^{\circ} \mathrm{C} / \mathrm{min}$, and $5{ }^{\circ} \mathrm{C} / \mathrm{min}$ under a $50 \mathrm{ml} / \mathrm{min}$ flowing argon atmosphere. Each DSC test was conducted using a Netzsch ${ }^{\mathrm{TM}}$ 404-C Pegasus DSC with a full temperature range calibration set and a typical baseline subtraction method. Resulting energetic curves were analyzed for endothermic phase transformation processes using the accompanying Proteus ${ }^{\mathrm{TM}}$ software package. Each solidus temperature measurement was resolved by using the onset temperature for the endothermic liquidus peak, which is determined by using the intercept of the upper slope tangent and the horizontal baseline. The corresponding liquidus temperature was resolved as the maximum endothermic peak value where no subsequent phase transformations occurred thereafter. Additional sub-liquidus transformation phenomena were identified by using maximum peak value measurements.

Endothermic peak characterization was confirmed by means of isothermal furnace heat treatment experiments with subsequent metallographic analysis. DSC results were also quantified in terms of solidification time (i.e. liquidus-solidus window), which indirectly can be used to characterize the degree of microsegregation.

\section{Thermodynamic Solidification Model}

In conjunction with the aforementioned experimental characterization methods, theoretical predictions were also obtained utilizing a computational thermodynamics approach. A phenomenological model was developed that can be used to predict the degree of chemical partitioning between the liquid and the $\gamma$-solid solution phases that occurs during alloy solidification for the 10 component species. The $\gamma$-solid solution and liquid phases are modeled by using a substitutional solution formalism with the aid of an extensive thermodynamic database constructed specifically for SX superalloy applications [11]. The thermodynamic lattice and excess mixing energy parameters for this streamlined multi-component model were derived from a more advanced multiphase model. This particular model, developed by Jaansalu of the Royal Military College of Canada, is capable of determining complete energetic transformation processes and has proven to be extremely useful and accurate in the calculation of equilibrium compositions and transformation temperatures of CMSX-10 and PWA-1484 turbine blade alloys [11]. With all the relevant binary and ternary interaction parameters finalized, the user database model parameters were entered into FACT-Sage ${ }^{\mathrm{TM}}$ software environment and solidification-microsegregation predictions were determined using the EQUILIB Gibbs-free energy minimization utility [15]. Each nominal alloy composition was entered into the software and a series of thermodynamic solidification predictions were generated as determined by the solid-liquid phase equilibria model.

\section{Results and Discussion}

\section{$\underline{\text { SX Casting Process }}$}

Provided in Figure 3 is a partial longitudinal cross-section of one successful casting, illustrating the transition from the large dendritic structure of the seed material to the start of the new region of SX growth.

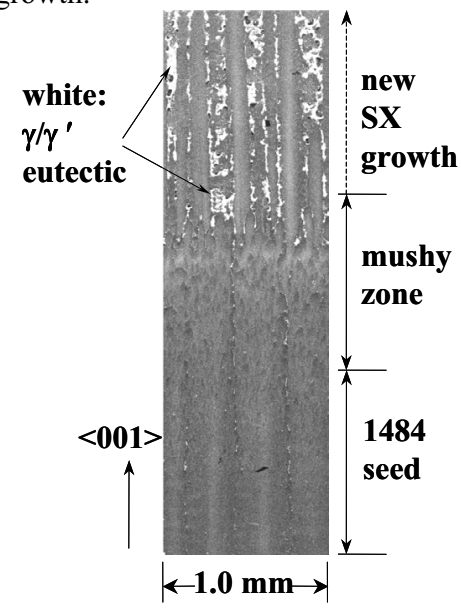

Figure 3: Partial longitudinal cross-section of bottom-end of cast bar, illustrating transition from the large dendritic structure of seed material, through the partially melted mushy zone, into the new region of SX growth. Large white areas are interdendritic $\gamma / \gamma^{\prime}$ eutectic. 
Apparent in the longitudinal micrograph is the typical dendritic cross-section, heavily decorated with a large fraction of $\gamma / \gamma^{\prime}$ eutectic located between the secondary dendrite arms. All six alloy compositions revealed an excellent SX solidification structure, with a common primary dendrite arm spacing of $\sim 250$ $\mu \mathrm{m}$. The SEM images provided in Figure 4 illustrate the uniformly distributed 3-dimensional structure of the SX dendrite morphology for both the transverse and longitudinal directions respectively. It should be noted that the interdendritic $\gamma-\gamma^{\prime}$ regions in these images are not present so that the dendritic structure can be more easily represented.

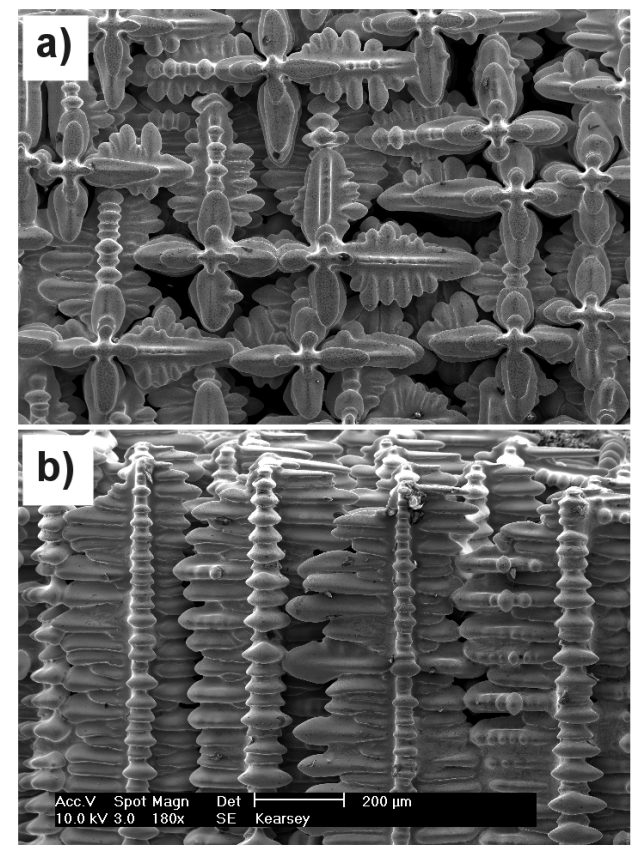

Figure 4: SEM image of uniformly distributed 3-D structure of the SX dendrite morphology for both (a) transverse and (b) longitudinal directions. Interdendritic liquid phase is not present.

\section{EPMA Microsegregation Results}

Chemical Segregation: Provided in Table II are the average EPMA compositional results for Alloys A through F. Although it is not reported here, the Sigma-1 variation for each element has been determined for all analysis points, indicating a very repeatable and accurate process. The degree of microsegregation between the dendrite cores and interdendritic regions can be better characterized with the use of the chemical segregation term, $k^{\prime}$. By definition, $k^{\prime}$ is taken to be the ratio of the dendrite core composition over the interdendritic composition level. The average segregation coefficient for each alloy has also been recorded in Table II. From these results, it is apparent that the typical segregation behavior of most SX superalloy systems is present [6]. Elements such as $\mathrm{Cr}, \mathrm{Co}, \mathrm{Re}, \mathrm{Mo}$, and $\mathrm{W}$ all tend to segregate towards the dendrite core regions during solidification, as indicated by the greater than unity $k^{\prime}$ values. In other words, these elements are the first to solidify within the cores during the SX withdrawal process. Elements that segregate in a contrasting manner have $k^{\prime}$ values less than unity, such as the $\gamma^{\prime}$ forming elements of $\mathrm{Al}, \mathrm{Ti}$, and $\mathrm{Ta}$. These elements are present in greater quantity in the last liquid phase to solidify and result in large eutectic $\gamma-\gamma^{\prime}$ phases to form within the interdendritic regions. Other elements that do not favorably segregate to either region have $k^{\prime}$ values close to unity and represent the ideal segregation behavior sought after by material designers. For the alloy compositions examined here, this would include the elements $\mathrm{Ni}$ and $\mathrm{Ru}$. Controlling the extent of this segregation behavior is critical to SX microstructural stability, as high levels of Re, W, $\mathrm{Cr}$, and Mo within the dendrite cores are known to promote TCP phase formation [10]. The formation of these deleterious phases in high temperature turbine applications results in premature creep properties, due to the resulting depletion of solid solution strengthening elements from the alloy matrix and also because of their brittle nature.

Upon examining the $k^{\prime}$ in Table II, it can be seen that Alloys A and $\mathrm{F}$ appear to have the lowest degree of chemical segregation of the six compositions examined. Alloys B, C, D and E all show extensive segregation of $\mathrm{Re}, \mathrm{W}, \mathrm{Mo}$ and $\mathrm{Cr}$ to the dendrite core regions as commonly observed in commercial alloys $[9,16,17,18$, 19]. The maximum and minimum $k^{\prime}$ coefficients for the alloys investigated are provided at the bottom of Table II, illustrating the degree of microsegregation variance. From the microsegregation values reported, it is apparent that elements such as $\mathrm{Al}, \mathrm{Ti}, \mathrm{Ni}, \mathrm{Co}$, $\mathrm{Ta}$, and $\mathrm{Ru}$ all segregate with approximately the same behavior based on their $k^{\prime}$ values. The lack of deviation of $k^{\prime}$ for $\mathrm{Al}$ and Ti may be explained by the narrow range of nominal levels of these elements among the alloys examined. However, Ni, Co and $\mathrm{Ta}$ levels vary considerably in the nominal compositions, yet they yield relatively consistent segregation coefficients. There does not seem to be any synergistic effects on segregation within these species due to element-element interactions or multi-element interactions based on the $k^{\prime}$ values for the six alloys examined. Conversely, elements such as $\mathrm{Re}, \mathrm{W}, \mathrm{Cr}$ and Mo seem to have varying segregation properties with respect to nominal composition levels.

Of particular interest in the EPMA microsegregation results is the superior segregation behavior of Alloy F. Not only does this alloy have a higher total refractory content than most of the alloys, but it also contains a greater amount of segregation-prone elements. It appears that the addition of $4 \mathrm{wt}$. \% Ru plays a major role in counteracting the segregation behavior of elements such as $\mathrm{Cr}, \mathrm{Re}$ and Mo. This can be clearly determined from Figure 5 showing the drastic decrease in preferential segregation in Alloy $F$ as compared to all five of the other alloys.

Alloys A through D all show an increase in chemical segregation as the level of Re is increased from 1 to $4 \mathrm{wt} \%$. Despite Alloys A to $\mathrm{D}$ containing the same $21 \mathrm{wt} . \%$ total refractory content, the $\mathrm{Cr}$, $\mathrm{Re}$ and Mo segregation coefficients increase from alloy A to D, consistent with the increasing Re content from Alloy A to D and despite the concurrent decreasing $\mathrm{W}$ content. Therefore, it appears that $\mathrm{Re}$ has a much greater influence on segregation behavior compared to W. Alloy E shows even stronger core segregation with the presence of both high $\mathrm{Re}$ and $\mathrm{W}$ content (i.e. greater refractory content). However, Alloy F, which has the same total refractory level of Alloy E, shows remarkable improvement in overall microsegregation. The lower segregation coefficient of Alloy $\mathrm{F}$ is not due to the lower W content compared to Alloy E, since Alloy F exhibits a lower partitioning coefficient than Alloy $\mathrm{C}$, which contains the same $\mathrm{W}$ level. 
Table II: Average EPMA compositions (wt.\%) for dendrite core and interdendritic $\gamma-\gamma^{\prime}$ with corresponding k' coefficients.

\begin{tabular}{lcccccccccc}
\hline Alloy-Region & $\mathrm{Ni}$ & $\mathrm{Cr}$ & $\mathrm{Co}$ & $\mathrm{Mo}$ & $\mathrm{W}$ & $\mathrm{Ta}$ & $\mathrm{Re}$ & $\mathrm{Al}$ & $\mathrm{Ti}$ & $\mathrm{Ru}$ \\
\hline A-core & 64.83 & 7.62 & 5.24 & 1.66 & 3.47 & 9.95 & 1.24 & 4.68 & 0.75 & 0.00 \\
A-interdendritic & 64.99 & 5.41 & 4.40 & 1.27 & 1.52 & 14.14 & 0.63 & 5.49 & 1.27 & 0.00 \\
B-core & 64.09 & 7.72 & 5.38 & 1.71 & 2.38 & 9.73 & 2.79 & 4.73 & 0.72 & 0.00 \\
B-interdendritic & 64.64 & 3.96 & 4.05 & 0.86 & 0.41 & 16.85 & 0.73 & 5.98 & 1.54 & 0.00 \\
C-core & 63.34 & 7.90 & 5.33 & 1.75 & 1.46 & 10.07 & 4.20 & 4.59 & 0.75 & 0.00 \\
C-interdendritic & 64.64 & 3.75 & 3.98 & 0.81 & 0.37 & 16.77 & 1.05 & 5.94 & 1.50 & 0.00 \\
D-core & 63.93 & 7.67 & 5.41 & 1.78 & 0.00 & 9.59 & 5.91 & 4.62 & 0.69 & 0.00 \\
D-interdendritic & 65.60 & 3.55 & 3.95 & 0.75 & 0.00 & 17.21 & 1.16 & 5.94 & 1.57 & 0.00 \\
E-core & 61.01 & 7.61 & 5.58 & 1.64 & 5.31 & 9.32 & 3.97 & 4.54 & 0.69 & 0.00 \\
E-interdendritic & 65.39 & 2.82 & 4.02 & 0.51 & 1.50 & 17.25 & 0.66 & 6.28 & 1.49 & 0.00 \\
F-core & 59.49 & 8.06 & 5.33 & 1.85 & 1.52 & 8.52 & 4.60 & 4.40 & 0.70 & 3.91 \\
F-interdendritic & 60.37 & 6.10 & 4.44 & 1.55 & 0.81 & 13.67 & 2.07 & 5.13 & 1.28 & 3.80 \\
Segregation k' & & & & & & & & & \\
Alloy A & 1.00 & 1.41 & 1.19 & 1.30 & 2.29 & 0.70 & 1.99 & 0.85 & 0.59 & $\mathrm{n} / \mathrm{a}$ \\
Alloy B & 0.99 & 1.95 & 1.33 & 1.98 & 5.75 & 0.58 & 3.83 & 0.79 & 0.47 & $\mathrm{n} / \mathrm{a}$ \\
Alloy C & 0.98 & 2.11 & 1.34 & 2.17 & 3.96 & 0.60 & 4.00 & 0.77 & 0.50 & $\mathrm{n} / \mathrm{a}$ \\
Alloy D & 0.97 & 2.16 & 1.37 & 2.39 & $\mathrm{n} / \mathrm{a}$ & 0.56 & 5.07 & 0.78 & 0.44 & $\mathrm{n} / \mathrm{a}$ \\
Alloy E & 0.93 & 2.70 & 1.39 & 3.22 & 3.55 & 0.54 & 5.99 & 0.72 & 0.46 \\
Alloy F & 0.99 & 1.32 & 1.20 & 1.19 & 1.87 & 0.62 & 2.22 & 0.86 & 0.55 \\
min k' & 0.93 & 1.32 & 1.19 & 1.19 & 1.87 & 0.54 & 1.99 & 0.72 & 0.44 & 1.03 \\
max k' & 1.00 & 2.70 & 1.39 & 3.22 & 5.75 & 0.70 & 5.99 & 0.86 & 0.59 & 1.03 \\
\hline
\end{tabular}

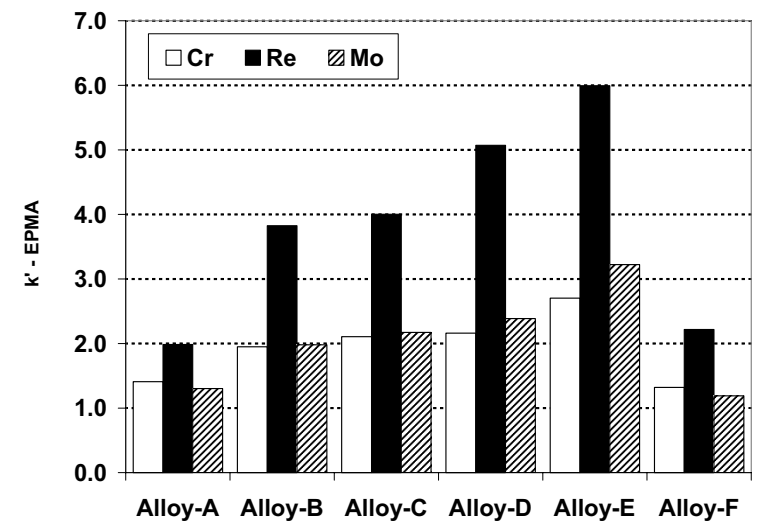

Figure 5: Measured segregation behavior of $\mathrm{Cr}$, $\mathrm{Re}$ and Mo. Alloys A-E show increased microsegregation with elevated nominal Re levels. Alloy F shows decreased segregation as a result of an additional 4 wt.\% Ru.

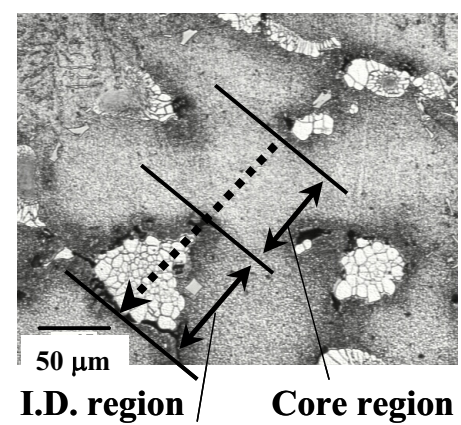

Figure 6: Transverse cross-section illustrating EPMA line-scan direction across dendritic structure.

Compositional Gradient: As previously mentioned, additional EPMA line scans were performed on each alloy along the major crystallographic directions in order to characterize the extent of chemical segregation during dendritic growth. It was observed that along both secondary arm directions there were negligible differences in localized compositions. In other words, there was no distinct compositional gradient present from the dendrite core region to the dendrite tips for each of the alloys examined. However, a sharp compositional gradient existed in each alloy when line scans were performed in the diagonal direction across the dendrite cores and through the neighboring interdendritic regions, as illustrated in Figure 6.
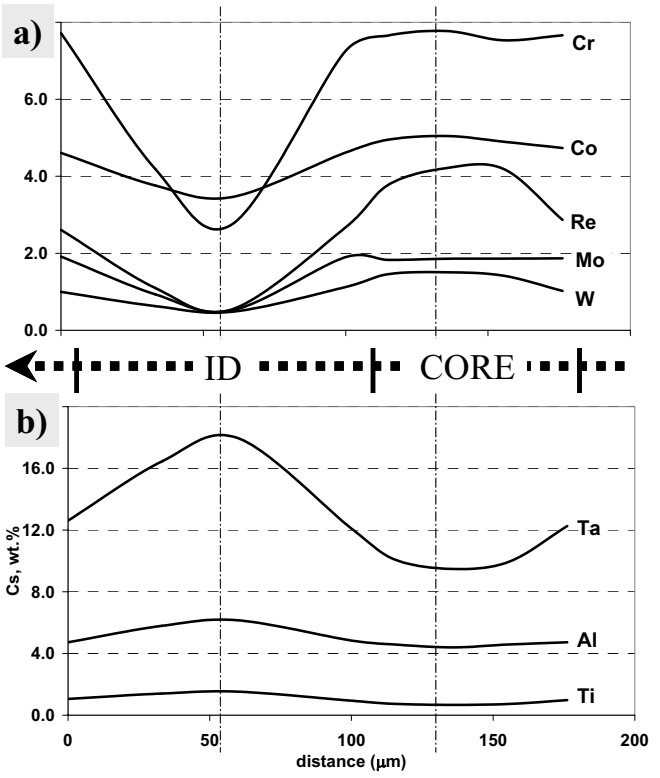

Figure 7: Results from EPMA line-scan for a) core elements and b) interdendritic elements.

Provided in Figure 7 are the results for a diagonal line scan performed on Alloy C. Those elements that tend to segregate to 
the dendrite core regions have been plotted in Figure 7(a); likewise, the compositional gradient for the interdendritic prone elements have been plotted in Figure 7(b). It is apparent that within the boundaries of the dendrite core, the composition for each element is relatively uniform. Upon approaching the edge of the core, sharp gradients in chemical composition are evident with a relatively uniform rate of change until the center of the interdendritic region is reached. This non-uniform chemical distribution within the interdendritic region seems to indicate that there exists a finite solidification time required for $\gamma-\gamma^{\prime}$ eutectic solidification. In other words, liquid-solid transformation is not immediate and hence true eutectic solidification does not occur upon traversing the eutectic transformation temperature.

\section{DSC Analysis and Thermo-Physical Characterization}

As previously mentioned, representative samples from each alloy were analyzed at thermal heating and cooling rates of $20^{\circ} \mathrm{C} / \mathrm{min}$, $10{ }^{\circ} \mathrm{C} / \mathrm{min}$, and $5{ }^{\circ} \mathrm{C} / \mathrm{min}$ under a $50 \mathrm{ml} / \mathrm{min}$ flowing argon atmosphere. One example of the recorded DSC energetic curves is provided in Figure 8 for Alloy-F in the as-cast condition. Upon heating, the first endothermic peak, $\mathrm{T}_{0}$, is associated with the initial interdendritic solutioning of the $\gamma^{\prime}$ phase. The second endothermic reaction, identified as $T_{1}$, is related to the $\gamma-\gamma^{\prime}$ eutectic transformation temperature. It should be mentioned that this particular value is barely noticeable for the heating rates of $20^{\circ} \mathrm{C} / \mathrm{min}$ and $10^{\circ} \mathrm{C} / \mathrm{min}$. However, better resolution is attained at the slower rate of $5^{\circ} \mathrm{C} / \mathrm{min}$ due to less significant super-heating effect. The $T_{1}$ values are resolved for the two faster rates by inspection of the $1^{\text {st }}$ derivative curves in each case. Finally, the values for $T_{S}$ and $T_{L}$ correspond to the solidus and liquidus points of each alloy for each particular DSC heating rate. For the most part, the reported liquidus temperatures are very reliable, as the values are characterized from the maximum endothermic peak temperatures as determined by the Proteus analysis software. On the other hand, the extrapolated solidus temperatures, as determined by the tangent-onset method, provide for a much larger range of operator error $\left(\sim 2-3^{\circ} \mathrm{C}\right)$. In an attempt to reduce this unavoidable human error, additional data processing was completed to find zero-heating rate transformation temperatures by extrapolating the data of all three heating rates.

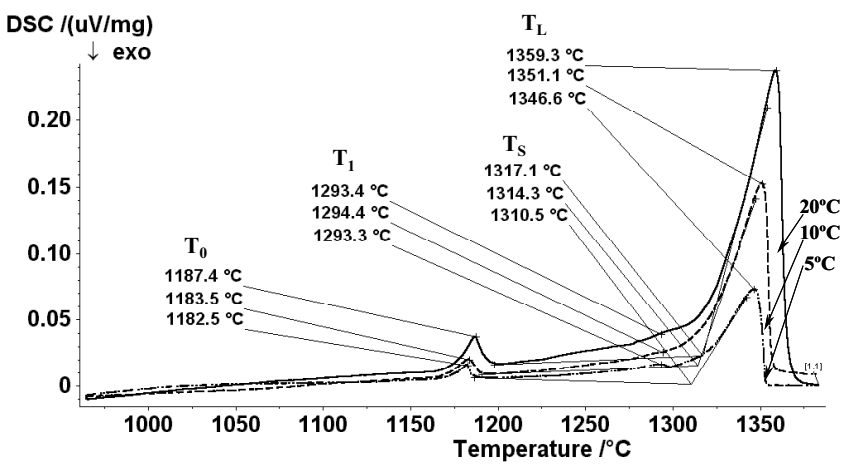

Figure 8: DSC energetic curves for as-cast Alloy-F at heating rates of 5,10 and $20{ }^{\circ} \mathrm{C} / \mathrm{min}$.

The DSC heating results for each alloy were analyzed in a similar manner as described above for Alloy F. The temperature transformation results were then plotted as a function of heating rate for all six alloy compositions. A linear-fit was then made for each set of data points and the extrapolated intercept temperature at $0{ }^{\circ} \mathrm{C} / \mathrm{min}$ was calculated to remove any heating rate effects. These corrected heating-rate values were determined for $T_{0}, T_{1}, T_{S}$ and $\mathrm{T}_{\mathrm{L}}$. An example of this extrapolation method has been provided for $\mathrm{T}_{\mathrm{L}}$ as shown in Figure 9.

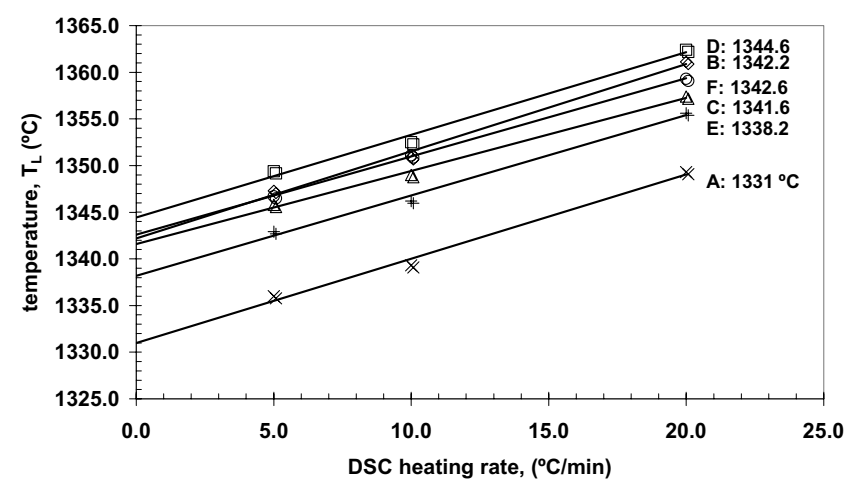

Figure 9: $\mathrm{T}_{\mathrm{L}}$ Extrapolation from DSC heating rate results.

Table III: Transformation temperatures $\left({ }^{\circ} \mathrm{C}\right)$ determined using heating rate extrapolation.

\begin{tabular}{lcccccc}
\hline Alloy & $\mathbf{T}_{\mathbf{0}}$ & $\mathbf{T}_{\mathbf{1}}$ & $\mathbf{T}_{\mathbf{S}}$ & $\mathbf{T}_{\mathbf{L}}$ & $\mathbf{T}_{\mathbf{L}}-\mathbf{T}_{\mathbf{S}}{ }^{*}$ & rank \\
\hline \hline Alloy A & 1184 & 1295 & 1303 & 1331 & 28 & 1 \\
Alloy B & 1173 & 1314 & 1310 & 1342 & 32 & 2 \\
Alloy C & 1187 & 1301 & 1310 & 1342 & 32 & 2 \\
Alloy D & 1182 & 1312 & 1312 & 1345 & 33 & 3 \\
Alloy E & 1172 & 1306 & 1303 & 1338 & 35 & 4 \\
Alloy F & 1180 & 1292 & 1310 & 1343 & 32 & 2
\end{tabular}

A summary of the extrapolated values is provided in Table III. Also recorded in Table III are the solidus-liquidus temperature ranges for each alloy. These $T_{L}-T_{S}$ values represent the temperature solidification range for each alloy during which solidliquid diffusion processes occur. In general, the greater the solidification temperature range (i.e. the longer it takes for the alloy to solidify completely), the higher the severity of microsegregation effects. In theory, shorter solidification times should result in less solute redistribution across the solid-liquid interface. Perfect chemical homogeneity could be achieved via liquid quenching methods, however, single crystal stability requirements and Bridgman furnace temperature gradients command a much slower growth rate. Hence, the lower $\Delta \mathrm{T}\left(\mathrm{T}_{\mathrm{L}^{-}}\right.$ $\mathrm{T}_{\mathrm{S}}$ ) values provided in Table III should indicate less severe microsegregation behavior. With this in mind, each alloy is ranked as a function of $\Delta \mathrm{T}$. The results predict that Alloy $\mathrm{A}$ should exhibit the least amount of microsegregation $\left(\Delta \mathrm{T}=28^{\circ} \mathrm{C}\right)$ with Alloy $\mathrm{E}$ having the highest predicted segregation severity $\left(\Delta \mathrm{T}=35^{\circ} \mathrm{C}\right)$. Alloys $\mathrm{B}, \mathrm{C}$ and $\mathrm{F}$ all have the same ranking as they were determined to have a $\Delta \mathrm{T}$ range of $32{ }^{\circ} \mathrm{C}$. Alloy $\mathrm{D}$ was predicted to have a slightly higher degree of microsegregation than Alloys $\mathrm{B}, \mathrm{C}$ and $\mathrm{F}$ as denoted by the $\Delta \mathrm{T}$ value of $33^{\circ} \mathrm{C}$, generally agreeing with the EPMA results of Figure 5.

\section{Eutectic Phase Fraction Analysis}

An example of the image analysis process used is provided in Figure 10 for Alloy C. For each alloy, 6 to 10 images were analyzed using different areas of the sample. The image magnification and analysis area was kept constant for each set of images. Lower magnification images would most likely yield better statistical results. However, the ability to differentiate 
between eutectic and dendritic areas was drastically reduced below the magnification chosen.

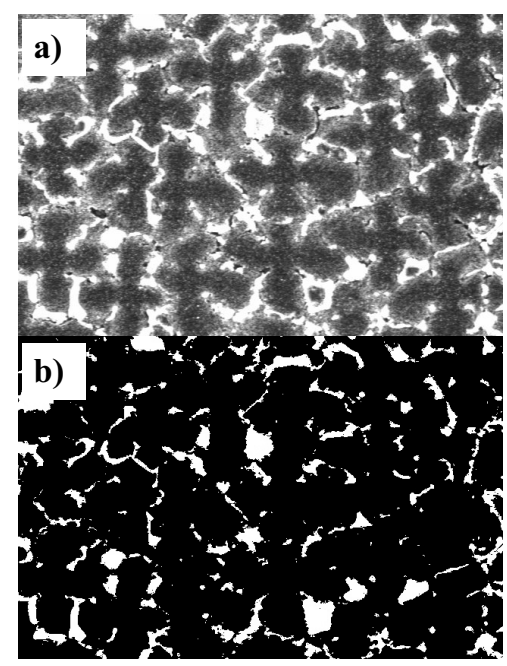

Figure 10: Transverse cross-section of Alloy C, a) etched optical image, b) black \& white threshold image used to determine the fraction of eutectic phase present.

Once a black and white threshold image is produced that clearly separates the dendritic and eutectic regions, a pixel counting subroutine is used to quantify the number of black and white (eutectic) pixels. For each image, the number of black and white pixels was measured with the corresponding fractions recorded. The fraction of eutectic phase present in the image was approximated by dividing the number of white pixels in the image by the total number of black and white pixels present. The eutectic fraction results are summarized in Table IV with respect to corresponding nominal wt.\% of $\mathrm{Re}, \mathrm{W}$, and $\mathrm{Ru}$ content. It can be seen from these results that for Alloys A through D, as the level of $\mathrm{Re}$ content is increased (corresponding decrease in $\mathrm{W}$ level) the eutectic phase fraction rises significantly. The maximum eutectic fraction, $17 \%$, is present for Alloy E, which contains both high levels of Re and $\mathrm{W}$ nominally. In fact, if the results for Alloy $\mathrm{C}$ and Alloy E are compared, it is apparent that there is an associated $4 \%$ increase in eutectic phase fraction due to the additional $3 \mathrm{wt} . \%$ $\mathrm{W}$ that is added in Alloy E.

Table IV: Eutectic fraction as a function of nominal Re, $\mathrm{W}$, and $\mathrm{Ru}$ composition

\begin{tabular}{cccccc}
\hline as-cast & \% eutectic & Std. Dev. & wt.\% Re & wt.\% W & wt.\% Ru \\
\hline \hline Alloy-A & 6.47 & 0.95 & 1 & 3 & 0 \\
Alloy-B & 6.99 & 1.01 & 2 & 2 & 0 \\
Alloy-C & 13.32 & 1.15 & 3 & 1 & 0 \\
Alloy-D & 16.13 & 2.45 & 4 & 0 & 0 \\
Alloy-E & 17.16 & 2.01 & 3 & 4 & 0 \\
Alloy-F & 11.31 & 1.32 & 3 & 1 & 4
\end{tabular}

Hence, for Alloy E, the additional increase in eutectic phase fraction when compared to Alloy $\mathrm{C}$ is due to either the elemental effects of $\mathrm{W}$ or due to the higher overall total refractory content (i.e. more eutectic present because of $23 \mathrm{wt} . \%$ total refractory in Alloy E compared to the $21 \mathrm{wt}$. \% for Alloy C). In any case, based on this comparison, it can be concluded that the increasing fraction of eutectic phase for Alloys A through D is due to the increasing level of Re content of each alloy and not due to the decreasing $\mathrm{W}$ content. A comparison can also be made between Alloy $\mathrm{C}$ and Alloy $\mathrm{F}$ where there appears to be a decrease in eutectic phase fraction, from $13 \%$ to $11 \%$. This reduction in eutectic fraction may be due to the addition of $4 \mathrm{wt} . \% \mathrm{Ru}$ in Alloy $\mathrm{F}$ or possibly due to the $1 \mathrm{wt} . \%$ lower Ta level

The above comparison of eutectic phase fraction is made with respect to the nominal composition levels for each alloy. It may also be useful to compare the eutectic levels of each alloy with respect to the actual interdendritic eutectic compositions measured in prior EPMA studies. Below in Table V is a list of the eutectic phase fraction and associated interdendritic EPMA compositions for each alloy.

Table V: Eutectic fraction as a function of localized interdendritic eutectic phase composition.

\begin{tabular}{cccccc}
\hline as-cast & \% eutectic & wt.\% Cr & wt.\% Al & wt.\% Ti & wt.\% Ta \\
\hline \hline Alloy-A & 6.47 & 5.41 & 5.49 & 1.27 & 14.14 \\
Alloy-B & 6.99 & 3.96 & 5.98 & 1.54 & 16.85 \\
Alloy-C & 13.32 & 3.75 & 5.94 & 1.50 & 16.77 \\
Alloy-D & 16.13 & 3.55 & 5.94 & 1.57 & 17.21 \\
Alloy-E & 17.16 & 2.82 & 6.28 & 1.49 & 17.25 \\
Alloy-F & 11.31 & 6.10 & 5.13 & 1.28 & 13.67
\end{tabular}

Figure 11 illustrates these results in the form of an area graph. Based on the information plotted, some preliminary findings can be stated. The graph clearly shows an increase in eutectic fraction as the wt.\% level of eutectic Al increases. This behavior can also be seen in Alloy F, as there is an associated drop in the eutectic fraction curve, which coincides with a drop in Al level. The same pattern can also be seen to some degree for the eutectic Ti level. Alloys $\mathrm{A}$ and $\mathrm{F}$ show decreased levels of $\mathrm{Ti}$ in the eutectic composition that may be related to their lower eutectic fraction levels. These findings do not seem to be unrealistic, as it is generally known that both $\mathrm{Al}$ and $\mathrm{Ti}$ strongly promote $\gamma^{\prime}$ precipitation, which is one of the main constituents in the $\gamma-\gamma^{\prime}$ eutectic phase. Another observation that can be made is the associated increase in eutectic fraction as a function of rising $\mathrm{Ta}$ level in the eutectic composition. Once again, Alloy F shows a decrease in eutectic fraction with a corresponding decrease in $\mathrm{Ta}$ level. This may be due to Ta substituting for $\mathrm{Ti}$ or $\mathrm{Al}$ in the $\mathrm{Ni}_{3} \mathrm{Al}-$ $\mathrm{Ti}$ structure. Finally, another interesting finding is the inverse effects of interdendritic $\mathrm{Cr}$ content on the fraction of eutectic present. Figure 11 clearly shows that, as the level of $\mathrm{Cr}$ content decreases for Alloys A through E, there is an inverse increase in relative eutectic fraction. This is further validated by observing the similar high $\mathrm{Cr}$ - low eutectic relationship present for Alloy F.

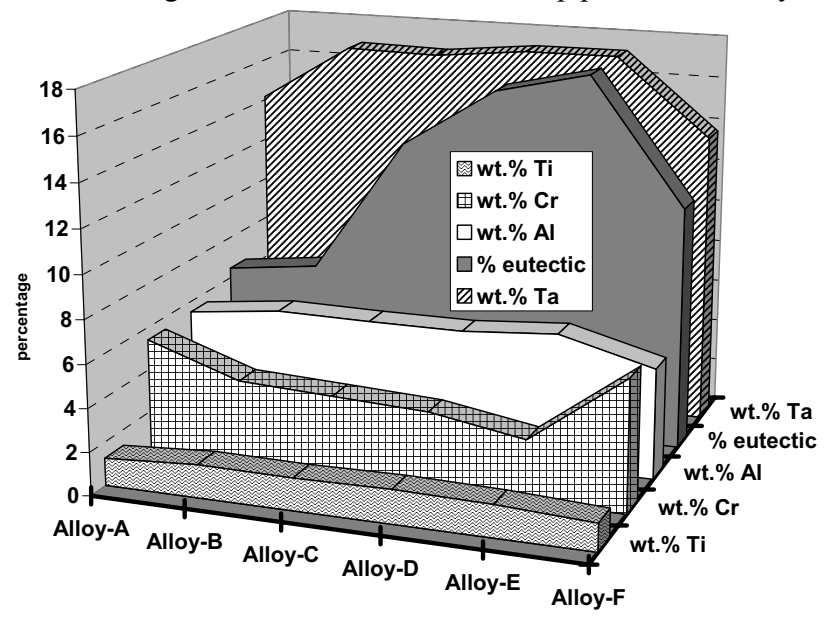

Figure 11: Comparison of eutectic phase fraction and associated eutectic composition levels. 
At this point, it should be mentioned that the effects of individual concentration levels on eutectic fraction can only be discussed. In reality, it is most likely that the synergistic effects of two or more interacting atomic species influence eutectic levels. Nonetheless, the following preliminary microsegregation findings can be presented:

1. Eutectic fraction increases with nominal Re content

2. W level does not appear to play a direct role in eutectic levels

3. Increased total refractory appears to increase eutectic fraction

4. Ru appears to decrease the overall eutectic fraction

5. Increasing interdendritic $\mathrm{Al}, \mathrm{Ti}$, and $\mathrm{Ta}$ levels result in an increase in eutectic fraction

6. Decreasing interdendritic $\mathrm{Cr}$ results in an increase in eutectic.

\section{Thermodynamic Solidification Predictions}

Each nominal alloy composition was entered into the software and a series of thermodynamic solidification predictions were generated as determined by the solid-liquid phase equilibria model. Utilizing the minimum Gibbs energy module, FACTEQUILIB, the compositions of the first solid to form and the last liquid to solidify were calculated for each alloy. In theory, these two compositions should represent the chemistry of the dendrite cores and interdendritic $\gamma-\gamma^{\prime}$ phases respectively. An example of these predictions has been plotted in Figure 12 and Figure 13 for Alloy A. In general, the model composition predictions for each alloy are reasonably close to the measured EPMA values. There does exist some degree of error with respect to the predicted $\mathrm{W}$ levels that may require further CALPHAD analysis of the $\mathrm{W}-\mathrm{X}$ binary systems. Using the same concept of $k^{\prime}$ that was presented in Figure 5 for the Cr-Re-Mo EPMA results, the same $k^{\prime}$ calculations were performed on the FACT compositional results. In a similar manner, Figure 14 is presented for the FACT $k^{\prime}$ results. These results clearly do not correspond with the EPMA $k^{\prime}$ values for $\mathrm{Cr}, \mathrm{Re}$, and Mo shown in Figure 5. In fact, based on the graph, the model results seem to indicate that Alloy F yields the poorest microsegregation results of all the alloys, with respect to $\mathrm{Re}, \mathrm{Cr}$, and Mo segregation. The difference in predicted and measured $k^{\prime}$ values may be due to non-equilibrium cooling effects or diffusional kinetic effects not accounted for in thermodynamic equilibrium calculations.

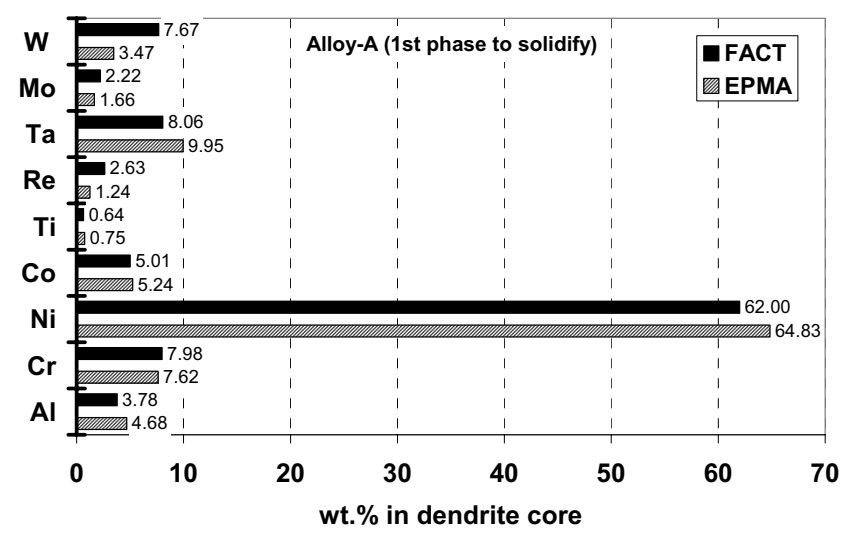

Figure 12: Model prediction for the composition of the $1^{\text {st }}$ solid phase to occur upon solidification (dendrite core).

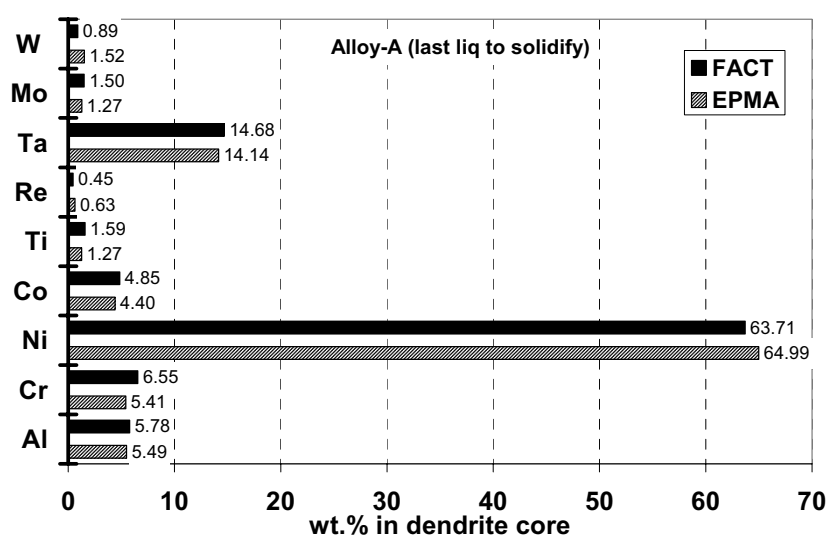

Figure 13: Model prediction for the composition of the last liquid to solidify (interdendritic region).

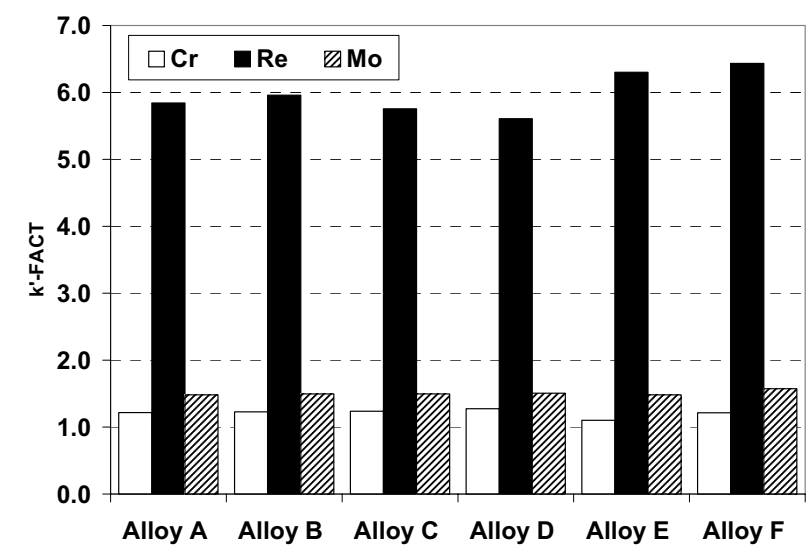

Figure 14: Model predicted segregation of Cr, Re and Mo.

A Gulliver-Scheil treatment was also generated for each alloy utilizing the thermodynamic solidification model. The segregation behavior for all 10 components was mapped as a function of each alloy's solidification temperature range. In this case, the segregation coefficient, $k$, is defined as $k_{\mathrm{i}}=\mathrm{X}_{\mathrm{i}}^{\mathrm{S}} / \mathrm{X}_{\mathrm{i}}^{\mathrm{L}}$ for the solid and liquid phases on either side of the solid-liquid solidification interface. Figure 15 through Figure 17 illustrate the $k$ segregation behavior for Re, Mo, and Ta respectively. From Figure 15 it is evident that the predicted partitioning behaviour for Re does not vary much with temperature, as indicated by the slightly U-shaped trend for each curve. However, there is a noticeable deviation in $k_{\mathrm{Re}}$ due to differences in nominal alloy composition, which range from $\sim 2.3$ for Alloy D to $\sim 2.6$ for Alloy F. It should be mentioned, however, that this range of $k$ is relatively small and does not resemble the EPMA measured segregation behavior, which indicates a large increase in Re segregation with increased nominal levels of Re in the base alloys. Figure 17 indicates that Ta segregation is more sensitive to temperature effects as indicated by the sharp rises in $k_{\mathrm{Ta}}$ over the plotted solidification range. For the most part, Ta does not demonstrate major differences in segregation as a consequence of nominal alloy levels, as designated by the densely packed $k$ curves. The predicted $k_{\mathrm{Mo}}$ results behave in a similar manner to that of Ta except for the noticeable difference in segregation of Alloy $\mathrm{F}$, indicating a possible negative effect of $\mathrm{Ru}$ addition on Mo segregation. 


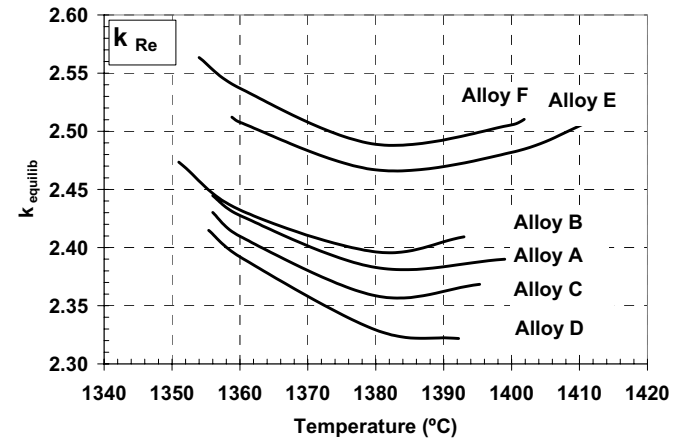

Figure 15: Model segregation results for Re.

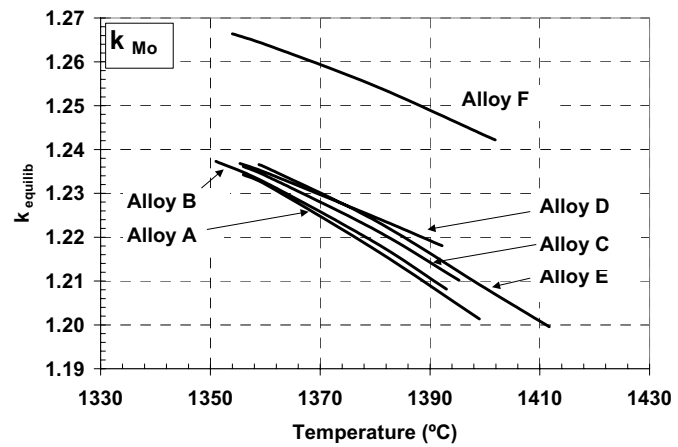

Figure 16: Model segregation results for Mo.

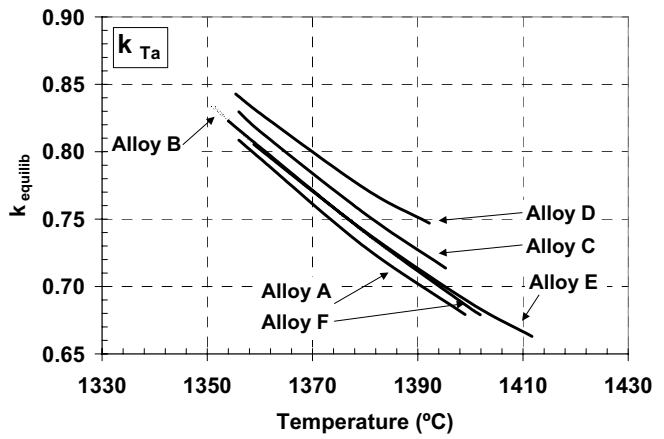

Figure 17: Model segregation results for Ta.

\section{Summary and Conclusions}

Increasing nominal Re levels amplified levels of microsegregation with respect to the dendrite core segregating elements of $\mathrm{Mo}, \mathrm{Cr}$, and, more significantly, Re. Higher total refractory contents resulted in increased microsegregation behavior. $\mathrm{Ru}$ additions effectively reduced the severity of microsegregation that occurs due to elevated Re and high total refractory levels.

For the alloys examined, as-cast eutectic fraction levels increase with nominal Re content. The nominal W level does not appear to play a direct role in eutectic levels. Increased total refractory level appears to increase the final as-cast eutectic fraction. Ru additions appear to decrease the overall eutectic fraction.

In comparison to microsegregation results determined via EPMA and eutectic phase fraction measurements, the $\Delta \mathrm{T}$ values qualitatively agree to a certain extent. However, due to the rather small differences in predicted solidification ranges and the likelihood of human error in measuring $T_{S}$ onset values from the DSC plots, microsegregation tendencies should not be based solely on extrapolated DSC $\Delta \mathrm{T}$ results.

A computational thermodynamic model was used in FACT to investigate the effects of nominal alloy levels on microsegregation. The typical trends in dendrite core and interdendritic segregation were predicted, as well as fairly accurate compositional predictions of the first solid to form and last liquid to solidify. A comparison of the partitioning trends between the six alloys did not reproduce the measured EPMA values. This may be remedied by combining the existing thermodynamic model with a kinetic diffusion model, accounting for microsegregation effects.

Based on EPMA, eutectic phase fraction and DSC results, the addition of $\mathrm{Ru}$ was responsible for a reduction in both dendritic and ID segregation. The addition of Ru may be responsible for the reduction in eutectic fraction, as well as the smaller $T_{L}-T_{S}$ window. This should result in easier homogenization and therefore improved phase stability for the SX alloy system investigated.

\section{References}

1. R.M. Kearsey et al., "Processing and Properties of Single Crystal NiBase Superalloys" (Report LTR-ST-SMPL-2003-0109, IAR-NRC, 2003).

2. G. Erickson, J Metals 1995; 47 (4): p.36-39.

3. M. Gell, In: Japan--U.S. Seminar on Superalloys. Susono, Japan: Japan Inst. of Met., 1984. p.457-473.

4. A.F. Giamei, D.L. Anton., Metall Trans A 1985; 16A (11): p.1997.

5. D. Blavette, P. Caron, T. Khan, In: Reichman S et al, Eds. Superalloys 1988. Warrendale, USA: TMS, 1988. p.305-314.

6. M. Durand-Charre, The Microstructure of Superalloys. Amsterdam, The Netherlands: Gordon and Breach Science Pub, 1997. p.60.

7. W. Du, et al. Chinese J Aeronautics 1998; 11 (1): p.61-65.

8 . R.M. Kearsey et al., "Compositional Design Considerations for Microsegregation in Single Crystal Superalloy Systems", Journal of Intermetallics, in press, submitted May 2003.

9. MSA Karunartne, CMF Rae, RC. Reed, Met \& Mat Trans A 2001; 32A (6): p.2409-2421.

10. F. Darolia, DF Lahrman, RD Field, In: Reichman S et al, Eds. Superalloys 1988. Warrendale, USA: TMS, 1988. p.255.

11. K.M. Jaansalu, "A Thermodynamic Model for Ni-based SX Gas Turbine Alloys”, PhD. Thesis, November 2002, Royal Military College of Canada

12. F. Ritzert et al., In: Processing of Metals and Advanced Materials: Modeling, Design, and Properties. San Antonio, TX: TMS, 1998, p.163174.

13. P. Caron, In: Pollock TM et al, Eds. Superalloys 2000, Warrendale, USA: TMS, 2000. p.737-746.

14. A. Didier et al., In: Pollock TM et al, Eds. Superalloys 2000, Warrendale, USA: TMS, 2000. p.829-838.

15. C.W. Bale, A.D. Pelton, W.T. Thompson, Facility for the Analysis of Chemical Thermodynamics, McGill Univ.-Ecole Polytechnique, 1996.

16. SN. Tewari et al., Tech Report: NASA-TM-103518, 1990. p.1.

17. R. Overfelt, Tech Report: AD-a384 209, Auburn Univ Al Space Power Inst, 2000. p.98.

18. Q, Feng et al., Acta Mat 2003; (51): p.269-284.

19. GE. Fuchs, Mater Sci Eng A 2001; A300: p.52-60. 